\title{
Mudança no perfil de tráfego de redes brasileiras em decorrência da pandemia pelo novo coronavírus
}

\author{
Allan Rodrigo de Souza Braga ${ }^{1}$, Diego $\operatorname{Passos}^{1}$ e Antonio Augusto de Aragão Rocha ${ }^{1}$ \\ ${ }^{1}$ Instituto de Computação - Universidade Federal Fluminense (UFF) \\ 24.210-310 - Niterói - RJ - Brasil
}

\begin{abstract}
Due to coronavirus pandemic, the traffic characteristics of computer networks, including the internet, registered a significant change. While the increase in internet traffic was evident, other networks, such as the LANs for corporations and research institutions, have showed a reduction in bandwidth consumption. However, this change in traffic profile is not limited to bandwidth consumption. There was also a change in the traffic type. In this work, we analyze this profile change under several different perspectives. In particular, an online opinion survey was carried out, aiming to analyze the profile of internet use from the users' point of view. The research focused on the intensity of internet use and the activity for which it was used, as well as on the user's perception of the connection stability. We also analyze the changes in the traffic patterns of Points-of-Presence of RNP (Rede Nacional de Ensino e Pesquisa), a network focused on higher education, research and innovation, and found out an increase of up to five times in the bandwidth consumption for videoconferencing applications, although the total bandwidth consumption has decreased.
\end{abstract}

Resumo. Em decorrência da pandemia pelo novo coronavírus, as características do tráfego das redes de computadores, incluindo a internet, registraram significativa mudança. Enquanto o incremento no tráfego da internet foi patente, outras redes, como as LANs para corporações e instituições de pesquisa, apontaram uma redução no consumo de banda. No entanto, a mudança no perfil de tráfego das redes não se limita ao consumo de banda. Também foi observada uma mudança no tipo de tráfego. Neste trabalho, analisamos essa mudança de perfil sob diversas perspectivas. Em particular, foi realizada uma pesquisa de opinião online, com o objetivo de analisar o perfil de utilização da Internet sob o ponto de vista dos usuários. O enfoque da pesquisa foi a intensidade de uso da internet e a atividade para a qual ela foi empregada, bem como a percepção do usuário sobre a estabilidade da conexão. Também analisamos as mudanças nos padrões de tráfego dos Pontos de Presença (PoPs) da RNP (Rede Nacional de Ensino e Pesquisa), voltada para a educação superior, pesquisa e inovação, e constatamos um aumento de até cinco vezes no consumo de banda para aplicações de videoconferência, embora o consumo total de banda tenha diminuído.

\section{Introdução}

O novo Coronavírus foi isolado, por autoridades Chinesas, em 7 de janeiro de 2020, de acordo com o Situation Report $1^{1}$. A COVID-19, doença causada pelo novo Coronavírus, foi caracterizada como pandêmica, pela Organização Mundial da Saúde (OMS), em 12 de 
março de 2020, em consonância com o Situation Report $52^{1}$. Desde então, considerando dados acumulados até novembro de 2020, as infecções por esse vírus, em todo o mundo, ultrapassaram a marca dos 46 milhões de casos, enquanto mais de 1,2 milhão de mortes foram notificadas, segundo o consignado no Weekly epidemiological update - 3 November $2020^{1}$.

Os alarmantes números da pandemia exigiram que os governos adotassem medidas emergenciais para tentar controlar a proliferação do vírus, reduzindo a contaminação ${ }^{2}$. Uma dessas medidas foi o isolamento da população. Cada país lidou de forma diferente com o isolamento. No Brasil, não houve diretrizes uniformes e cada estado / município estabeleceu seus indicadores e ações decorrentes, como o funcionamento de apenas serviços essenciais, estabelecido no Rio de Janeiro e São Paulo. China, Irã, Coréia do Sul, Estados Unidos da América e países europeus, como Itália, Espanha, França e Reino Unido, adotaram medidas de isolamento social mais rígidas.

As medidas de isolamento reduziram a circulação de pessoas e, por conseguinte, a transmissão do vírus. No entanto, efeitos colaterais podem ser observados, em especial nas características de tráfego das redes, como a Internet. Como exemplo, as chamadas de voz e vídeo no WhatsApp e no Facebook Messenger tiveram um acréscimo de mais de $100 \%^{3}$; Segundo o vice-presidente corporativo do Microsoft 365, a empresa registrou, no aplicativo Microsoft Teams, um aumento de 500\% nas reuniões, chamadas e conferências, e um aumento de $200 \%$ no uso em dispositivos móveis ${ }^{4}$. Já a Netflix e o YouTube decidiram reduzir a qualidade do streaming na Europa para evitar o colapso das redes, devido à demanda sem precedentes ${ }^{5}$. No Brasil, o IX.br registrou um aumento de $25 \%$ no tráfego no primeiro trimestre de 2020 e, devido à pandemia, foi alcançado o pico de $10 \mathrm{~Tb} / \mathrm{s} \mathrm{em}$ 23 de março ${ }^{6}$. Esse aumento de tráfego refletiu a mudança na rotina das pessoas, como a redução da mobilidade (Figura 1a), a maior concentração das pessoas em casa (Figura 1b) e a maneira como elas se comunicam, trabalham, estudam, consomem serviços e realizam compras.

Para analisarmos as mudanças no padrão de tráfego e no perfil de uso da Internet, bem como de outras redes, como as LANs para corporações e instituições de pesquisa, realizamos uma pesquisa de opinião. O enfoque foi na intensidade de uso da internet, nas atividades para as quais ela foi empregada pelo usuário, bem como na percepção do usuário sobre a estabilidade da conexão. Considerando que a percepção das pessoas não reflete, necessariamente, com precisão, o comportamento das redes, complementamos esse estudo com a análise do tráfego de aplicações de conferência/videoconferência e dos Pontos de Presença (PoPs) de uma rede de grande escala, a RNP (Rede Nacional de Ensino e Pesquisa). O objetivo foi entender como essas mudanças ocorreram e o impacto que elas causaram.

Este trabalho está organizado da seguinte forma: na Seção 2 será avaliado o per-

\footnotetext{
${ }^{1}$ https://www.who.int/emergencies/diseases/novel-coronavirus-2019/situation-reports

${ }^{2} \mathrm{http}$ ///associacaopaulistamedicina.org.br/noticia/isolamento-e-quarentenas-como-paises-estaolidando-ao-redor-do-mundo

${ }^{3}$ https://edition.cnn.com/2020/03/18/tech/zuckerberg-facebook-coronavirus-response/index.html

${ }^{4}$ https://www.microsoft.com/en-us/microsoft-365/blog/2020/03/05/our-commitment-to-customersduring-covid-19/

${ }^{5}$ https://edition.cnn.com/2020/03/19/tech/netflix-internet-overload-eu/index.html

${ }^{6}$ https://cgi.br/noticia/releases/ix-br-reaches-mark-of-10-tb-s-of-peak-internet-traffic/
} 
fil de uso da Internet, através de uma pesquisa de opinião; na Seção 3 serão elencadas e caracterizadas algumas ferramentas de comunicação, bem como sua evolução; na Seção 4 será apresentada uma análise de tráfego das principais ferramentas multimídia de comunicação; na Seção 5 será analisado o tráfego da Rede Nacional de Ensino e Pesquisa (RNP), dos meses de março, abril, junho, julho e agosto de $2020^{7}$, referentes a 15 estados brasileiros (AC, AL, AM, AP, ES, MA, MS, MT, PB, PI, RN, RO, RR, SE e TO); na Seção 6 serão discutidos os trabalhos relacionados; e na Seção 7 apresentamos as conclusões do nosso estudo.

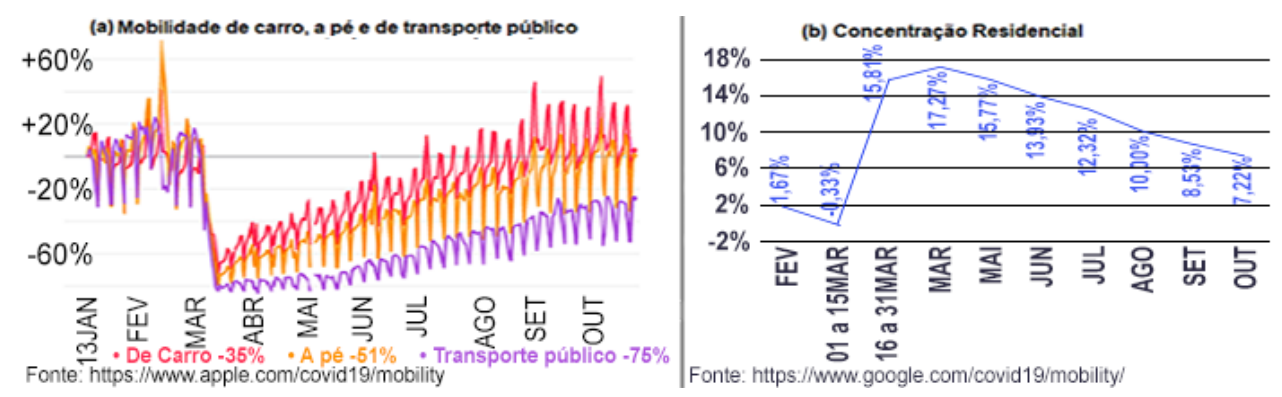

Figura 1. Variação da mobilidade no Brasil

\section{Análise do perfil de uso da Internet pelos Usuários}

Para entender melhor a mudança no perfil de utilização da internet, em virtude da pandemia pelo novo Coronavírus, foi realizada uma pesquisa de opinião. A proposta da pesquisa foi a análise de variáveis quantitativas, no que tange aos aplicativos utilizados, e variáveis qualitativas, no que se refere à estabilidade da conexão de Internet. Foi aplicada a estatística descritiva [Guimarães 2008] a fim de identificar as informações mais relevantes acerca das variáveis pesquisadas, possibilitando o cruzamento de informações, objetivando a obtenção de inferência estatística. Para viabilizar a pesquisa, foi elaborado um questionário eletrônico, utilizando a ferramenta gratuita Google Forms. A pesquisa ${ }^{8}$ ficou disponível do dia $1^{\circ}$ de junho de 2020 ao dia 13 de junho de 2020.

O link para a pesquisa foi distribuído em grupos de WhatsApp e Telegram da comunidade acadêmica da Universidade Federal Fluminense (UFF), grupos de trabalho, de amigos, da família e de moradores da cidade de Niterói-RJ, além de publicado em páginas de redes sociais. No período em que a pesquisa ficou ativa, 379 pessoas, em 11 estados brasileiros, na faixa etária de 9 a 76 anos, responderam ao questionário. A única limitação do escopo populacional da pesquisa foi quanto ao país (Brasil), sendo utilizada uma amostra aleatória.

\subsection{Análise de Dados}

Os resultados da pesquisa demonstram que 105 pessoas $(27,7 \%)$ aumentaram a intensidade de uso da internet com fins profissionais, enquanto $33(8,7 \%)$ reduziram e 241 $(63,6 \%)$ mantiveram a intensidade. A quantidade de pessoas que utilizam a internet com muita intensidade, para trabalho, passou de 155 (40,9\%) para $230(60,7 \%)$, durante a pandemia. Isso representa um aumento de $48,4 \%$.

\footnotetext{
${ }^{7}$ Em virtude de dificuldades técnicas, não foi possível obter acesso aos dados do mês de maio de 2020.

${ }^{8} \mathrm{https} / / /$ docs.google.com/forms/d/e/1FAIpQLSetF4deZrFdqMPapvU3MUNQd5oVMdP18YJdCINK_yrhFg $1 \mathrm{xg} /$ closedform
} 
Observa-se, ainda, que 96 pessoas $(25,3 \%)$ aumentaram a intensidade de uso da internet para estudo, enquanto 34 (9\%) reduziram e 249 (65,7\%) mantiveram a intensidade. A quantidade de pessoas que utilizam a internet com muita intensidade, para estudo, passou de $141(37,2 \%)$ para $206(54,4 \%)$, durante a pandemia, um aumento de 46,1\%. Foi possível, também, verificar que 98 pessoas $(25,9 \%)$ aumentaram a intensidade de uso da internet também para lazer e/ou entretenimento, enquanto $27(7,1 \%)$ reduziram e 254 (67\%) mantiveram a intensidade. A quantidade de pessoas que utilizam a internet com muita intensidade, para lazer e/ou entretenimento, passou de $206(54,4 \%)$ para $273(72 \%)$, durante a pandemia, representando um aumento de 32,5\%. Assim, identifica-se um expressivo aumento na intensidade de utilização da internet, que é similar para todos os tipos de utilização (trabalho, estudo e lazer), variando entre $25,3 \%$ e $27,7 \%$.

No entanto, a utilização da internet com muita intensidade registrou, nesta pesquisa, aumento entre $32,5 \%$ e $48,4 \%$. Esse aumento foi muito próximo para as atividades de trabalho e estudo, entre $46,1 \%$ e $48,4 \%$. Para a atividade de lazer e entretenimento, o aumento foi menor, embora expressivo, atingindo o índice de 32,5\%. O aumento menor se deve ao fato de que esta atividade já era muito demandada anteriormente. Assim, as atividades de lazer e/ou entretenimento continuam sendo as atividades mais demandadas, com muita utilização relatada por $72 \%$ dos respondentes. Por outro lado, as atividades de trabalho e estudo registram o índice de $60,7 \%$ e $54,4 \%$, respectivamente. Releva destacar que o tráfego relacionado ao lazer e/ou ao entretenimento, normalmente, é aquele com o maior consumo banda. Seu emprego, em geral, está associado aos tráfegos mais densos, como streaming de vídeo e jogos online.

Uma informação importante que pode ser obtida com a análise dos dados coletados pela pesquisa é que, após a decretação da pandemia, $47 \%$ dos participantes passou a trabalhar em regime de tele trabalho, seja em regime integral ou parcial, o que certamente colaborou para o aumento do tráfego registrado no Brasil ${ }^{9}$. $\mathrm{O}$ aumento do tráfego também pode ter influenciado a percepção sobre a estabilidade da conexão com a internet. Aqueles que relataram que utilizavam uma conexão com a internet estável e, após a decretação da pandemia, essa conexão passou a apresentar instabilidades são 39\%. No entanto, ao somar a esse indicador à quantidade de pessoas que relataram que já utilizavam uma conexão com a internet instável, mesmo antes da pandemia, tem-se a indicação de que $61,5 \%$ das pessoas enfrentam dificuldades para terem acesso à rede mundial de computadores.

No que se refere à percepção sobre a estabilidade da conexão com a internet, foi observada a ausência de correlação com a quantidade de dispositivos conectados à internet: a quantidade de respondentes que relataram conexão estável para residências onde há de 1 a 5 dispositivos conectados mantém equilíbrio com aquelas residências onde há de 6 a 10 dispositivos conectados. O mesmo equilíbrio foi constatado para o cenário de conexão instável.

Em relação aos aplicativos de mensagens instantâneas, a pesquisa revelou que quase a totalidade dos respondentes utiliza o WhatsApp $(99,7 \%)$. No entanto, para a função de videoconferência/chamada de vídeo, essa relação cai para 81,5\%, em que pese a manutenção da liderança e a alta taxa de utilização, seguido por: Zoom (56\%), Google

\footnotetext{
${ }^{9}$ https://cgi.br/noticia/releases/ix-br-reaches-mark-of-10-tb-s-of-peak-internet-traffic/
} 
Meet (37\%), Skype (28\%), Microsoft Teams (19\%), Facebook Messenger (13\%) e Cisco Webex $(8 \%)$.

\section{Aplicações para multimídia}

Um dos maiores impactos do isolamento social é o relacionamento interpessoal. Até então, esse relacionamento era estimulado a ser presencial. Isso porque há estudos que revelam que, no relacionamento interpessoal virtual, um indivíduo adquire bons e maus hábitos, podendo culminar na dependência de ambientes virtuais ou, até mesmo em uma fobia social [King et al. 2013].

Após a chegada da pandemia e necessidade de distanciamento social, a comunicação passou a ser prementemente virtual. Com isso, diversos aplicativos, dentre eles os de comunicação, tiveram um expressivo incremento em sua utilização, sendo abordados, neste estudo, os de mensagens instantâneas, de chamadas de voz, de conferências, de chamadas de vídeo e de videoconferências.

\subsection{Aplicativos de Mensagens Instantâneas}

As mensagens de texto têm sido usadas com sucesso na área de saúde. Em 2009 as mensagens de texto foram usadas para promover a aplicação da segunda e terceira dose da vacina do Papilomavírus Humano (HPV) [Kharbanda et al. 2011] e na prevenção contra o vírus Influenza [Szilagyi and Adams 2012]. Com o surgimento da COVID-19, as mensagens de texto ${ }^{10}$ também foram utilizadas para alertar a população sobre os perigos da doença e formas de prevenção ${ }^{11}$.

Nesse diapasão, a constante evolução das tecnologias permitiu uma comunicação muito mais rápida e sem custo adicional. As mensagens de texto tradicionais, como o Short Message Service (SMS), estão perdendo espaço, em especial entre os usuários de dispositivos móveis inteligentes, para os aplicativos de mensagens instantâneas, como o WhatsApp [Church and de Oliveira 2013]. Alguns aplicativos de mensagens instantâneas permitem a criação de chatbots [Hill et al. 2015], que podem representar uma importante ferramenta em alguns tipos de serviço. Nesse contexto, em abril de 2020, a OMS criou um serviço interativo, utilizando um aplicativo de mensagens instantâneas, para que a população possa tirar dúvidas sobre o novo coronavírus ${ }^{12}$. Embora os aplicativos de mensagens instantâneas sejam utilizados na comunicação informal, essa categoria de aplicativos pode intermediar comunicações complexas de trabalho, como agendamentos e reuniões [Isaacs et al. 2002].

Um dos aplicativos de mensagens instantâneas mais usados é o WhatsApp [O'Hara et al. 2014]. É comum, atualmente, que esse tipo de aplicativo ofereça recursos para o compartilhamento de mídia, como imagens, vídeos, áudios e até a localização atual do dispositivo [O'Hara et al. 2014]. A eficiência desses aplicativos, no que se refere a utilização de banda, foi analisada [Zhang et al. 2015] tomando-se como base o

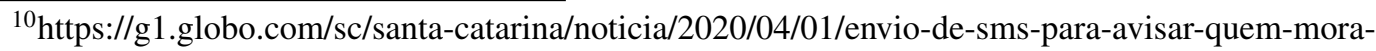
perto-de-pessoas-com-coronavirus-comeca-a-funcionar-em-florianopolis.ghtml

${ }^{11}$ https://www.sc.gov.br/noticias/temas/coronavirus/coronavirus-em-sc-governo-do-estado-ja-emitiumais-de-120-mil-alertas-em-sms-a-populacao-sobre-casos-de-covid-19

${ }^{12} \mathrm{https}$ ///nacoesunidas.org/oms-disponibiliza-numero-de-whatsapp-para-esclarecer-duvidas-sobre-acovid-19/
} 
tráfego gerado por caractere enviado / recebido. Dentre os aplicativos comparados (WeChat, WhatsApp, Facebook Messenger, Line e Viber), o Facebook Messenger obteve o pior desempenho, enquanto os melhores foram o WhatsApp e o Line. Esse mesmo estudo demonstrou que o desempenho dos aplicativos de mensagens instantâneas é inferior à de outros tipos de aplicações, como e-mail e navegação web, se atendo às mensagens de texto e não considerando os recursos que utilizam maior largura de banda, como imagens, vídeos e áudios.

\subsection{Chamadas de Voz/Vídeo e Videoconferências}

Com o passar do tempo, as tecnologias que possibilitam o tele trabalho têm evoluído significativamente. Dentre essas tecnologias, as chamadas de voz e vídeo e as videoconferências tornam o trabalho remoto viável [Jackson and van der Wielen 2002]. Em abril de 2020, o Governo Brasileiro publicou um documento para orientar trabalhadores e empregadores quanto aos cuidados que deveriam ser adotados no período da pandemia $^{13}$. Dentre esses cuidados, cita-se o de evitar reuniões presenciais. Essa orientação pode ter contribuído para que as reuniões passassem a ser majoritariamente virtuais, utilizando recursos de videoconferência. Dentre as ferramentas disponíveis para videoconferências, citam-se: WhatsApp, Skype, Google Meet, WebEx e GoToMeeting. Todas as ferramentas apresentadas têm arquitetura cliente-servidor. Dessas ferramentas, apenas o WhatsApp e o Skype oferecem, paralelamente, suporte à arquitetura Peer-to-Peer (P2P) [Gurung and Kim 2015].

Outro fator atrativo dessas ferramentas é que as chamadas de voz são baseadas em VoIP (Voice Over Internet Protocol). Uma de suas vantagens é custo, pois as ligações entre usuários da mesma plataforma, em geral, são gratuitas, sendo necessária apenas uma conexão de internet. Ao contrário da telefonia tradicional, o VoIP não exige a construção de uma infraestrutura exclusiva. A vantagem econômica é tão relevante, que há uma tendência para que as operadoras de telefonia substituam o atual sistema de comutação de circuito pelo VoIP [Seo 2008]. Diversos aplicativos de mensagens instantâneas oferecem o serviço de VoIP, tornando-o ainda mais popular.

Um debate importante sobre essas ferramentas diz respeito à segurança. Nesse quesito, sua utilização massiva, de forma repentina, não fugiu à regra. O aumento da utilização de meios eletrônicos e das redes, como a internet, se traduz em oportunidade para os cibercriminosos. Tendo em vista que o usuário representa, normalmente, o elo mais fraco da segurança da informação digital [da Silva and Stein 2011] e que, com o tele trabalho, a utilização de dispositivos computacionais residenciais, livres do controle organizacional, aumentou no período da pandemia, o risco do comprometimento de informações tende a aumentar.

Embora não tenha havido aumento significativo no número de ataques, cujo nível foi considerado moderado, a popularização das videochamadas introduziu uma nova fonte de vulnerabilidades no dia-a-dia dos usuários, segundo um estudo realizado por especialistas em segurança da Kaspersky ${ }^{14}$. Ainda de acordo com o mesmo estudo, observou-se, somente em relação ao Skype, um total de mais de 120 mil arquivos suspeitos disfarçados

\footnotetext{
${ }^{13}$ https://www.gov.br/pt-br/noticias/trabalho-e-previdencia/2020/04/ministerio-da-economia-lancamanual-de-orientacoes-para-empregadores

${ }^{14}$ https://www.kaspersky.com.br/blog/online-reunioes-ataques-hackers/14724/
} 
da aplicação original.

Na pesquisa realizada neste estudo, o aplicativo de videoconferência Zoom ocupa o segundo lugar entre os mais usados em sua categoria, com 56,5\% de adesão. No entanto, em consulta à base de dados do Common Vulnerabilities and Exposures $(\mathrm{CVE})^{15}$, foram encontrados, considerando apenas entradas do ano de 2020, registros de vulnerabilidades da seguinte ordem: uma crítica, sete altas e uma baixa. Em face disso, a utilização do aplicativo foi proibida pela Agência Nacional de Vigilância Sanitária (Anvisa), pela fabricante de foguetes SpaceX e pelas Forças Armadas Australianas ${ }^{16}$. A procuradoriageral de Nova York chegou a cobrar explicações a empresa desenvolvedora do aplicativo, citando, inclusive, um suposto compartilhamento de dados com o Facebook ${ }^{17}$. Contudo, utilizando o mesmo critério, destacam-se o Cisco Webex, cujos registros de vulnerabilidades são: uma crítica, oito altas, quatorze médias e quatro baixas e o WhatsApp com duas aguardando análise, três críticas, cinco altas, três médias e uma baixa. O Skype apresentou apenas uma vulnerabilidade crítica e duas médias. Não foram encontrados registros de vulnerabilidades em 2020 para o Facebook Messenger, Google Meet e para o Microsoft Teams. Com isso, observa-se que os aplicativos mais utilizados para videoconferência apresentam os maiores índices de registros de vulnerabilidades, o que leva à exposição de parcela significativa dos dispositivos.

Desse modo, é necessário que o ambiente computacional para o home office seja adequadamente protegido através de ferramentas de segurança, tais como firewall e antivírus [da Silva 2017] [Silva et al. 2013]. A segurança deve ser ainda maior se a rede local for sem fio.

\section{Análise de Tráfego das Aplicações Multimídia}

Para melhor entender as ferramentas de conferência e videoconferência mais utilizadas, foi realizada uma análise de tráfego utilizando somente áudio e outra análise utilizando áudio e vídeo. A amostra coletada foi de, aproximadamente, dois minutos e as configurações dos participantes das chamadas, conferências ou videoconferências foram um subconjunto da topologia apresentada na Figura 2 (A e C; B e C; ou A, B e C). Ressalta-se que $\mathrm{B}$ e $\mathrm{C}$ faziam parte da mesma rede local em sub-redes distintas.

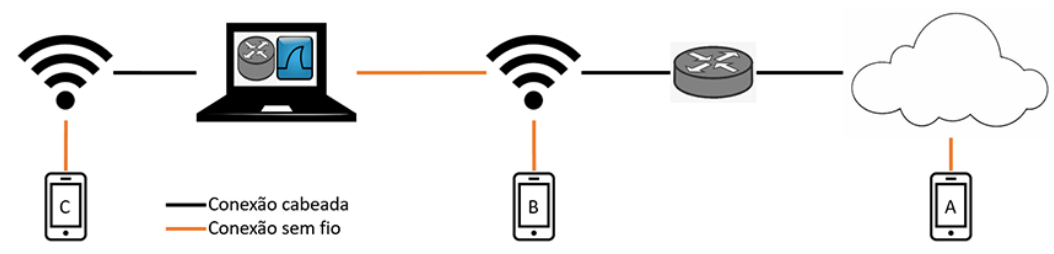

Figura 2. Topologia da rede de testes

$\mathrm{O}$ analisador de tráfego utilizado foi o Wireshark, instalado em notebook com sistema operacional Ubuntu Linux, que desempenhou, também, a função de roteador. A interface sem fio do notebook foi conectada ao roteador do ISP e a interface Ethernet

\footnotetext{
${ }^{15} \mathrm{https}: / / \mathrm{cve} . \mathrm{mitre} . \mathrm{org} /$

${ }^{16} \mathrm{https} / / / \mathrm{g} 1$.globo.com/economia/tecnologia/blog/altieres-rohr/post/2020/04/07/por-que-o-zoom-e-alvode-desconfianca-e-quais-sao-as-alternativas-para-videoconferencia.ghtml

${ }^{17}$ https://epocanegocios.globo.com/Empresa/noticia/2020/04/zoom-entra-na-mira-da-justica-de-novayork-por-problemas-de-seguranca.html
} 
conectada a um Ponto de Acesso (AP). A esse AP conectou-se o dispositivo monitorado pelo analisador de tráfego.

\subsection{Análise de Tráfego Utilizando Somente Áudio}

A análise de tráfego das ferramentas de comunicação, utilizando somente áudio, foi realizada com os dispositivos B e C, representados na Figura 2, para dois participantes, e utilizando os dispositivos A, B e C, ilustrados na mesma figura, para três participantes.

Consoante Figura 3a, observa-se que o incremento no consumo de banda na conferência com três participantes, tomando como base a chamada de voz com dois participantes, é mais expressivo no Google Meet e no Facebook Messenger, com um acréscimo de 106,13\% e 64,41\%, respectivamente. Já no Microsoft Teams houve um decremento de $8,68 \%$. As demais ferramentas mantiveram um acréscimo entre $5 \%$ e $27 \%$. O leve incremento no consumo de banda sugere que os servidores das respectivas aplicações mesclam os fluxos de áudio recebidos dos participantes [Hawwa 2002]. Assim, cada participante recebe apenas um fluxo de áudio do servidor.

A ferramenta que apresenta o maior consumo de banda é Zoom. Ela consome mais que o dobro que o Skype, segunda ferramenta que mais consome banda. A ferramenta que tem menor consumo de banda é o WhatsApp.

\subsection{Análise de Tráfego Utilizando Áudio e Vídeo}

A análise de tráfego das ferramentas de comunicação, utilizando áudio e vídeo, foi realizada com os dispositivos B e C, representados na Figura 2, para dois participantes, e utilizando os dispositivos A, B e C, ilustrados na mesma figura, para três participantes.

Conforme ilustrado na Figura 3b, o Facebook Messenger se fixa com o maior incremento de consumo de banda, nessa modalidade, com 41,8\%. As demais ferramentas mantiveram um acréscimo entre $18 \%$ e $33 \%$. A ferramenta que tem o maior consumo de banda é Google Meet e a que tem o menor consumo de banda é o WhatsApp, consumindo, com três participantes, menos da metade da banda consumida pelo Skype, segunda ferramenta de mais baixo consumo de banda.
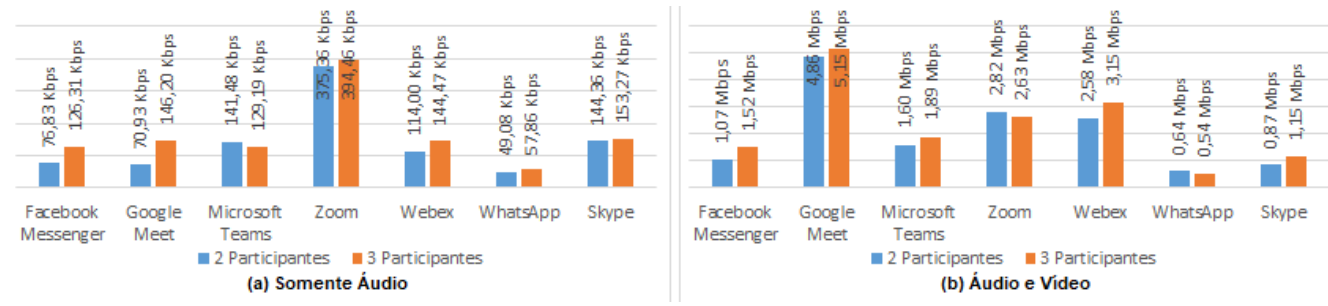

Figura 3. Gráficos comparativos de consumo de banda

Comparando os dados constantes das Figuras $3 a$ e $3 b$ verifica-se um comportamento distinto das ferramentas. Da chamada de vídeo, com dois participantes, para a videoconferência, com três participantes, o Google Meet, que registrou o maior aumento utilizando somente áudio, nesta modalidade é o que registra o menor aumento no consumo de banda, desconsiderando o WhatsApp e o Zoom, que registraram decréscimo de $15,8 \%$ e $6,64 \%$, respectivamente. Salienta-se que, sob certas condições, as aplicações podem manipular parâmetros de codificação, como a quantidade de quadros por segundo, para reduzir a utilização de banda. 


\subsection{Skype e WhatsApp}

Foi observada, nas ferramentas Skype e WhatsApp, durante a análise de tráfego, a capacidade de intercambiar do modo cliente-servidor para o modo par-a-par. Para chamadas com mais de dois participantes, as ferramentas utilizaram o modo cliente-servidor. $\mathrm{O}$ modo P2P está disponível apenas para chamadas com dois participantes.

Observou-se também que ambas as ferramentas conseguem detectar se a conexão P2P é local ou trafega pela internet. Ao concluir que a conexão P2P é local, as aplicações transmitem os fluxos de dados diretamente ao outro dispositivo utilizando o IP privado da rede local. O consumo de banda em cada um desses tipos de conexão é significativamente diferente. A taxa de transferência do Skype na rede local é $152 \%$ maior do que na internet e a taxa de transferência do WhatsApp é $232 \%$ maior na rede local, se comparado ao da internet.

\subsection{Round Trip Time (RTT)}

Para realizar a medição do Round Trip Time (RTT), foi calculado o tempo para o recebimento do pacote $S Y N-A C K$ após a transmissão do pacote $S Y N$, durante o Three-way Handshake da conexão TCP (Transmission Control Protocol), que é estabelecida pelas aplicações antes da transmissão dos fluxos de dados UDP (User Datagram Protocol). Foi calculada a média entre o valor obitido através do método descrito e do resultado de medições utilizando o Internet Control Message Protocol (ICMP), por intermédio do aplicativo ping.

As ferramentas de comunicação analisadas apresentaram RTT entre 13 ms e 26 ms, com exceção do Cisco Webex, que apresentou RTT de 182 ms. Considerando apenas o atraso de propagação, esses servidores estariam a uma distância máxima da cidade de Niterói-RJ, onde os experimentos foram realizados, entre $1.950 \mathrm{~km}$ e $3.900 \mathrm{~km}$. Por outro lado, ferramentas de geolocalização sugerem que os IPs desses servidores estejam localizados a distâncias muito maiores. Isso pode indicar que essas aplicações utilizam endereços IP anycast [Ma et al. 2009], ou seja, que implementam mecanismos para que os clientes possam selecionar o "melhor" servidor em um grupo de servidores. Já a distância máxima do servidor do Cisco Webex seria de $27.300 \mathrm{~km}$. A recomendação de limite de tempo aceitável para uma transmissão VoIP unilateral é de 150 ms [Goode 2002]. Destarte, o Cisco Webex é mais suscetível a problemas de interatividade para a localidade geográfica onde foram realizados os testes.

Embora não tenha sido possível determinar a quantidade exata de saltos para todos os servidores, encontrou-se um número mínimo de seis saltos.

\section{Análise de Dados do Backbone da RNP}

A Rede Nacional de Ensino e Pesquisa (RNP) é uma rede de abrangência nacional voltada para educação superior, pesquisa e inovação. Além de se conectar com 15 países da América Latina, com a rede europeia Géant e com os Estados Unidos, a RNP possui 27 Pontos de Presença (PoPs) no Brasil, um em cada estado da federação. Suas 50 redes comunitárias, mais de 4 milhões de usuários, 1.100 pontos e 800 organizações conectadas demonstram a relevância da RNP para nosso estudo.

Extraímos, dessa rede, no formato CSV (Comma-separated values), registros NetFlow com volume de aproximadamente 7 PB. Os dados foram filtrados em duas fases, 
uma por protocolo e outra por protocolo/portas ${ }^{18}$. Após isso, os dados receberam tratamento para identificação do PoP e foram cadastrados em um banco de dados. Os dados foram compilados por uma ferramenta de Business Intelligence (BI) desenvolvida pelos autores.

Foram analisados os dados dos meses de março, abril, junho, julho e agosto de 2020 referentes a 15 estados brasileiros (AC, AL, AM, AP, ES, MA, MS, MT, PB, PI, RN, RO, RR, SE e TO). Os demais estados não fizeram parte da análise uma vez que não havia dados disponíveis desde março de 2020, o que inviabilizaria o comparativo do tráfego pré e pós decretação da pandemia.

Assim como a internet, dados indicam que a Rede Nacional de Ensino e Pesquisa (RNP) também registrou uma mudança significativa no perfil do tráfego. No entanto, enquanto o tráfego da internet foi majorado, o tráfego da RNP reduziu. Pode-se observar na Figura 4 que o tráfego total do protocolo TCP foi reduzido em $73 \%$ do mês de março para abril de 2020. Nos meses de abril, junho, julho e agosto, o volume de tráfego total se manteve estável. No que tange às portas 80 e 443, principais portas utilizadas sobre o TCP, essas representam, em média, $90 \%$ do tráfego total. Uma possível justificativa para a redução do tráfego constatada é a redução e, em alguns casos, a suspensão das atividades presenciais nas Universidades.

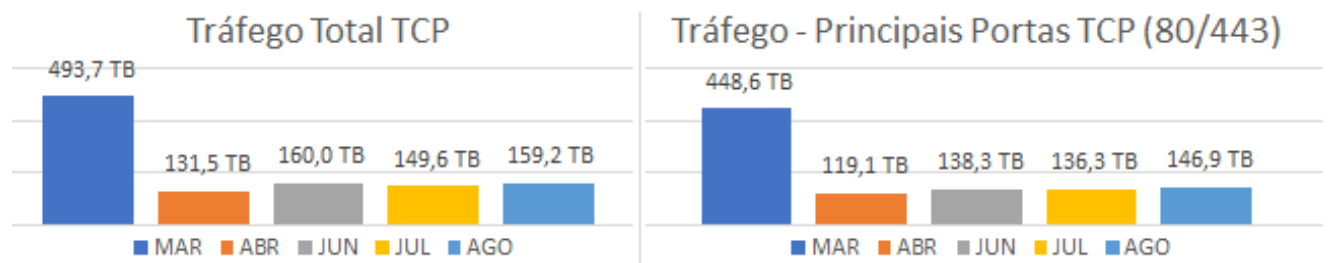
Figura 4. Gráficos comparativos de tráfego TCP - Total / Principais Portas
$(80 / 443)$

Como mostram as Figuras 4 e 5, a redução do tráfego total do protocolo UDP foi menor que a do TCP, com o índice de $42 \%$. Esse menor impacto pode estar relacionado ao fato de protocolos de gerência de redes, roteamento e outros, como o Simple Network Management Protocol (SNMP), Routing Information Protocol (RIP), Dynamic Host Configuration Protocol (DHCP) e Domain Name System (DNS), serem (majoritariamente) baseados no UDP. Além disso, a Figura 4 e a Figura 5 mostram que houve significativo acréscimo no tráfego das ferramentas de conferência / videoconferência analisadas nesse estudo (ferramentas e portas usadas): Facebook Messenger (40003), Google Meet (19305), MS Teams (3478, 3479, 3480, 3481), Skype (3478, 3479, 3480), Webex (9000), WhatsApp (3478) e Zoom (8801). Destaca-se que o Skype e o WhatsApp usam portas altas aleatórias no caso de chamadas entre apenas dois participantes, se utilizando conexão par-a-par. Assim, esse cenário não foi incluído na análise.

A queda no tráfego referente às aplicações de videoconferência, registrada apenas no mês posterior ao da decretação da pandemia, não foi proporcional à queda do tráfego total, sendo significativamente menor. $\mathrm{O}$ volume de tráfego dessas aplicações, nos meses posteriores, não acompanhou a tendência de estabilidade do volume de tráfego total do UDP, excetuado o mês de julho de 2020, onde houve um pico de tráfego $29 \%$ superior a

\footnotetext{
${ }^{18}$ TCP/UDP (40003, 19305, 3478, 3479, 3480, 3481, 9000, 8801, 80 e 443)
} 
média de tráfego dos meses de março a agosto de 2020 (Figura 5). Em vez disso, o tráfego referente às aplicações de videoconferência registrou expressivo aumento, com exceção do mês de abril de 2020, no qual houve uma readaptação da sociedade. Em agosto de 2020, o tráfego dessas portas foi quase o dobro daquele registrado antes da pandemia. O destaque no tráfego de videoconferência é registrado nas portas 8801 (utilizada pelo Zoom) e 19305 (utilizada pelo Google Meet), ambas chegando a quase 5 vezes de incremento no volume de tráfego, se comparados os meses março e agosto de 2020 (Figura $6)$.

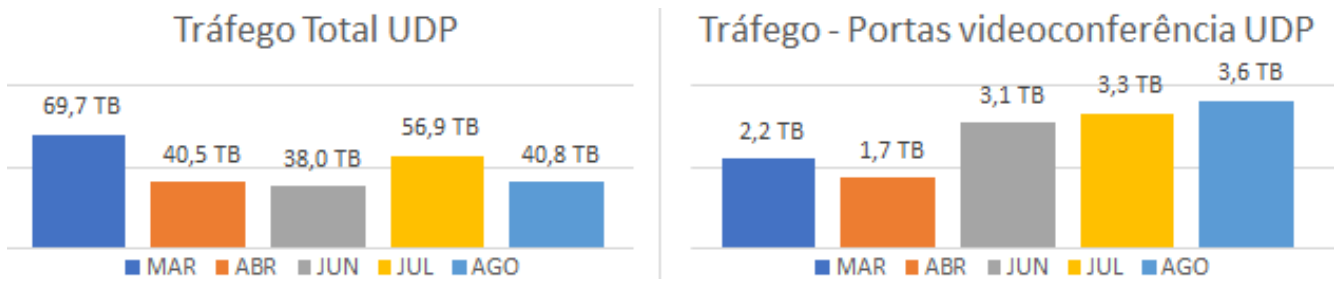

Figura 5. Gráficos comparativos de tráfego UDP - Total / Portas de videoconferência $(40003 / 19305 / 3478 / 3479 /, 3480 / 3481 / 9000 / 8801)$

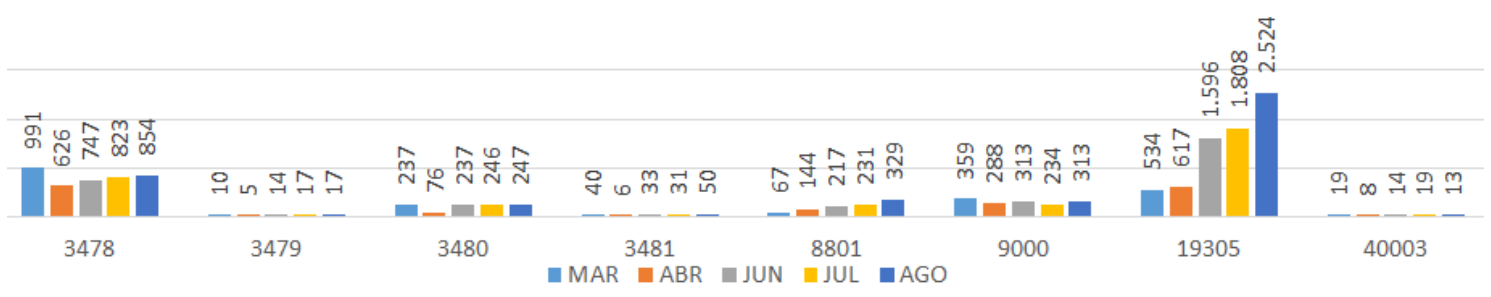

Figura 6. Gráfico de tráfego UDP das portas referentes a videoconferência (GB)

\section{Trabalhos Relacionados}

Outros trabalhos também analisaram o impacto da pandemia sobre o tráfego das redes de computadores com perspectivas complementares às deste estudo.

Em um trabalho sobre as implicações da pandemia da COVID-19 no tráfego da internet, [Feldmann et al. 2020] analisaram um conjunto de dados obtidos em um ISP da Europa Central, uma rede acadêmica metropolitana espanhola e 3 IXP — localizados na Europa central, no sul da Europa e na costa leste dos EUA. Os autores encontraram uma redução de $55 \%$ no volume de tráfego da rede acadêmica - uma redução maior que os $35 \%$ encontrados na nossa análise dos dados da RNP. Dados daquele trabalho também registraram um aumento de até $30 \%$ no tráfego dos IXP, após as medidas de restrição de mobilidade. Além disso, também foi notado um aumento significativo do tráfego de aplicações de videoconferência, em especial no horário comercial. O mesmo aconteceu com a porta TCP/993, usada pelo IMAP sobre TLS, e com a porta TCP/8200, usada por serviço de streaming de canais de TV russos. Com isso, os autores concluíram que o aumento foi relacionado a trabalho e entretenimento. Tal conclusão coaduna com os resultados da pesquisa de opinião deste estudo, onde verificou-se aumento na intensidade de uso da internet na ordem de $27,7 \%$ para trabalho, $25,3 \%$ para estudo e $25,9 \%$ para lazer e/ou entretenimento.

O estudo conduzido por [Böttger et al. 2020] sobre a reação da internet à COVID19 examinou a rede de borda do Facebook. É interessante observar o relato sobre a 
diferença na magnitude do impacto nas regiões do mundo. Para exemplificar, a América do Sul está elencada entre as regiões que experimentaram estresse em suas redes. Pudemos observar tal afirmação em nossa pesquisa de opinião, na qual 39\% dos respondentes relataram que passaram a observar uma instabilidade na conexão com a internet, que não existia anteriormente. Assim como em outros estudos, o trabalho em tela relata que o aumento do tráfego se dá, principalmente, em decorrência de conteúdo multimídia. Para embasar suas conclusões, os autores também analisaram a QoE (Quality of Experience) de vídeos, utilizando a métrica BSR (Bad Session Rate). Eles encontraram uma degradação da experiência do usuário e a correlacionaram com métricas de rede, como RTT e redirecionamento do tráfego para enlaces indiretos. Ao observar que todos os fatores coincidiam, concluíram que há a indicação do congestionamento de rede.

No Reino Unido, segundo [Lutu et al. 2020], a redução na mobilidade foi $50 \%$. Esse índice foi similar ao do Brasil (Figura 1) (em média, pouco mais de 50\%). O conjunto de dados do estudo foi proveniente de uma operadora de rede móvel do Reino Unido com suporte à $2 \mathrm{G}, 3 \mathrm{G}$ e $4 \mathrm{G}$. A informação curiosa apresentada pelo estudo é a redução do volume de tráfego de dados (downlink) da ordem de 25\%. Correlacionando essa redução com o período de medidas restritivas decorrentes da COVID-19, os autores inferiram que uma causa possível seria uma maior utilização da banda larga residencial. Já o uplink sofreu alterações mais modestas, entre $-7 \% \mathrm{e}+1,5 \%$, provavelmente em consequência do aumento do tráfego de voz sobre LTE (VoLTE), que atingiu um pico de $150 \%$, segundo os autores do artigo.

\section{Conclusões}

Neste artigo, foram estudados o perfil de utilização da Internet sob o ponto de vista dos usuários e as mudanças nas características do tráfego das redes de computadores, utilizando recursos de pesquisa de opinião e análise de tráfego dos PoPs da RNP e de aplicações multimídia.

Em virtude da pandemia, a relação das pessoas com a tecnologia mudou. A dependência de aplicações, como as de videoconferência e streaming de vídeo, aumentou, seja para atividades de lazer, estudo ou profissionais. Com isso, a infraestrutura de conexão com a internet foi mais demandada, enquanto houve uma tendência de redução nas redes corporativas e de instituições de ensino e pesquisa. O aumento da demanda pode ter contribuído para o elevado índice de pessoas que relataram dificuldades na conexão com a internet. Possíveis soluções são melhorias na infraestrutura da última milha [Greenstein 2016] e adoção maciça do padrão IEEE 802.11ax [Natkaniec et al. 2020].

Ficou evidenciado que as aplicações de videoconferência tiveram um aumento expressivo em sua utilização, conforme comprovado pela análise de tráfego da RNP, sendo um dos responsáveis pela mudança no perfil de utilização das redes. O impacto causado, na RNP, por exemplo, foi o incremento de 1,4TB/mês no tráfego, após cinco meses de utilização das ferramentas de videoconferência. Em verificação elaborada com base na Common Vulnerabilities and Exposures (CVE), foi constatado que algumas aplicações de videoconferência tiveram elevado número de vulnerabilidades identificadas apenas em 2020. O destaque é a ferramenta Cisco Webex, com 27 vulnerabilidades. Essa ferramenta também se destaca pelo elevado RTT, o que indica a possibilidade de que seja a única que não mantém servidor no Brasil, podendo degradar o desempenho da comunicação 
realizada através desse aplicativo.

O Skype e o WhatsApp se distinguem das demais ferramentas pela capacidade de intercambiar entre os modos cliente-servidor e par-a-par, dependendo do tipo de conexão dos dispositivos. Essa funcionalidade, se implementada pelos outros aplicativos, poderia reduzir a demanda sobre seus respectivos servidores e reduzir o tráfego na internet.

\section{Agradecimentos}

Agradecemos à Rede Nacional de Ensino e Pesquisa (RNP) pelo compartilhamento dos dados com a Universidade Federal Fluminense (UFF), que colaborou sobremaneira para a obtenção de resultados precisos, fomentando a pesquisa e desenvolvimento no país.

\section{Referências}

Böttger, T., Ibrahim, G., and Vallis, B. (2020). How the internet reacted to covid-19: A perspective from facebook's edge network. In Proceedings of the ACM Internet Measurement Conference, IMC '20, page 34-41, New York, NY, USA. Association for Computing Machinery.

Church, K. and de Oliveira, R. (2013). What's up with whatsapp? comparing mobile instant messaging behaviors with traditional sms. In Proceedings of the 15th international conference on Human-computer interaction with mobile devices and services (MobileHCI '13), pages 352-361, New York, NY, USA.

da Silva, D. R. P. and Stein, L. M. (2011). Segurança da informação: uma reflexão sobre o componente humano. Ciências \& Cognição, 10:46-53.

da Silva, R. R. (2017). Home-officer: um surgimento bem-sucedido da profissão pósfordista, uma alternativa positiva para os centros urbanos. Revista Brasileira de Gestão Urbana, 1(1):85-94.

Feldmann, A., Gasser, O., Lichtblau, F., Pujol, E., Poese, I., Dietzel, C., Wagner, D., Wichtlhuber, M., Tapiador, J., Vallina-Rodriguez, N., Hohlfeld, O., and Smaragdakis, G. (2020). The lockdown effect: Implications of the covid-19 pandemic on internet traffic. In Proceedings of the ACM Internet Measurement Conference, IMC '20, page 1-18, New York, NY, USA. Association for Computing Machinery.

Goode, B. (2002). Voice over internet protocol (voip). Proceedings of the IEEE, 90(9):1495-1517.

Greenstein, S. (2016). Congestion on the last mile. IEEE Micro, 36(6):62-63.

Guimarães, P. R. B. (2008). Métodos quantitativos estatísticos. IESDE Brasil S.A., 1st edition.

Gurung, S. and Kim, Y. (2015). Healthcare privacy: How secure are the voip/videoconferencing tools for phi data? In 2015 12th International Conference on Information Technology - New Generations, pages 574-579.

Hawwa, S. (2002). Audio mixing for centralized conferences in a sip environment. In Proceedings. IEEE International Conference on Multimedia and Expo, volume 2, pages 269-272 vol.2. 
Hill, J., Ford, W. R., and Farreras, I. G. (2015). Real conversations with artificial intelligence: A comparison between human-human online conversations and human-chatbot conversations. Computers in Human Behavior, 49:245-250.

Isaacs, E., Walendowski, A., Whittaker, S., Schiano, D. J., and Kamm, C. (2002). The character, functions, and styles of instant messaging in the workplace. In Proceedings of the 2002 ACM conference on Computer supported cooperative work (CSCW '02), pages 11-20, New York, NY, USA.

Jackson, P. and van der Wielen, J. (2002). Teleworking: New International Perspectives From Telecommuting to the Virtual Organisation. Management of technology and innovation. Taylor \& Francis.

Kharbanda, E. O., Stockwell, M., Fox, H., Andres, R., Lara, M., and Rickert, V. (2011). Text messaging to promote hpv vaccination. Journal of Adolescent Health, 48:S4-S5.

King, A., Valença, A., Silva, A., Baczynski, T., Carvalho, M., and Nardi, A. (2013). Nomophobia: Dependency on virtual environments or social phobia? Computers in Human Behavior, 29:140-144.

Lutu, A., Perino, D., Bagnulo, M., Frias-Martinez, E., and Khangosstar, J. (2020). A characterization of the covid-19 pandemic impact on a mobile network operator traffic. In Proceedings of the ACM Internet Measurement Conference, IMC '20, page 19-33, New York, NY, USA. Association for Computing Machinery.

Ma, Z., Zhou, J., and Zhang, L. (2009). A scalable framework for global application anycast. In 2009 Second International Conference on Information and Computing Science, volume 1, pages 250-253.

Natkaniec, M., Prasnal, L., and Szymakowski, M. (2020). A performance analysis of ieee 802.11ax networks. International Journal of Electronics and Telecommunications, vol. 66(No 1):225-230.

O'Hara, K. P., Massimi, M., Harper, R., Rubens, S., and Morris, J. (2014). Everyday dwelling with whatsapp. In Proceedings of the 17th ACM conference on Computer supported cooperative work \& social computing (CSCW'14), pages 1131-1143, New York, NY, USA.

Seo, S. (2008). Voip-telephone service: Economic efficiencies and policy implications. Telematics and Informatics, 25:47-55.

Silva, M. A., Ferreira, R., Leite, O., Macedo, S., and dos Santos, S. (2013). Segurança e confiabilidade para ambiente soho. HOLOS, 4(0):66-76.

Szilagyi, P. G. and Adams, W. G. (2012). Text Messaging: A New Tool for Improving Preventive Services. JAMA, 307(16):1748-1749.

Zhang, L., Xu, C., Pathak, P. H., and Mohapatra, P. (2015). Characterizing instant messaging apps on smartphones. In Mirkovic, J. and Liu, Y., editors, Passive and Active Measurement, pages 83-95, Cham. Springer International Publishing. 